\title{
Observer-Based Sliding Mode Load Frequency Control of Power Systems under Deception Attack
}

\author{
Siwei Qiao $\mathbb{D}^{1},{ }^{1}$ Xinghua Liu $\mathbb{D},{ }^{1}$ Gaoxi Xiao, ${ }^{2}$ and Shuzhi Sam $\mathrm{Ge}^{3}$ \\ ${ }^{1}$ School of Electrical Engineering, Xi'an University of Technology, Xi'an 710048, China \\ ${ }^{2}$ School of Electrical and Electronic Engineering, Nanyang Technological University, 639798, Singapore \\ ${ }^{3}$ Department of Electrical and Computer Engineering, National University of Singapore, 117583, Singapore \\ Correspondence should be addressed to Siwei Qiao; qswxut@163.com and Xinghua Liu; liuxh@xaut.edu.cn
}

Received 15 August 2021; Accepted 14 September 2021; Published 4 October 2021

Academic Editor: Chih-Chiang Chen

Copyright (C) 2021 Siwei Qiao et al. This is an open access article distributed under the Creative Commons Attribution License, which permits unrestricted use, distribution, and reproduction in any medium, provided the original work is properly cited.

This study investigates the observer-based sliding mode load frequency control for multiarea interconnected power systems under deception attack. By introducing the observer and combining it with the system state equation, the expression of the system error is obtained. A sliding mode surface is proposed to make sure the state of the systems to be stable. Then, the state equation of the system under sliding mode control is derived. The asymptotic stability of the whole system is proved by using the linear matrix inequality (LMI) technique and Lyapunov stability theory. Furthermore, a sliding mode control law is proposed to ensure that the attacked power system can reach a stable position. Numerical simulation results are presented to support the correctness of the results.

\section{Introduction}

The developments of the power systems have greatly facilitated the human life. Ensuring stability and sustainability of power systems, as an increasingly important research area, has attracted extensive attentions [1-3]. One of the most popular goals of such studies is to ensure stable operation of the power system, to which the use of control methods helps provide a boost $[4,5]$.

Sliding mode control, with its advantages in overcoming system uncertainties and achieving strong robustness against disturbances, has been widely used in a variety of fields [6-8]. System robustness is enhanced by combining dynamic surface control with the sliding mode [9-12]. It can be used in different systems such as nonlinear systems [13], Markovian jump systems [14], power systems, and so on. Here, we focus on the power system. The robust stability of adaptive event-triggered load frequency control (LFC) with sliding mode control (SMC) for multiarea power systems are investigated in [4]. The authors of [15] proposed a fractional-order sliding mode controller for effectively stabilizing a nonlinear power system in a fixed time. In [16], a highly robust observer sliding mode-based load frequency and tie-power control to compensate for primary frequency control of multiarea interconnected power systems were designed. While the power system can be kept stable by sliding mode control, the modern power system relies on the critical cyber infrastructure which makes it vulnerable to hostile cyber threats.

Some robust control methods are applied to the LFC of the multiarea power system. It should be pointed out that in the analysis of multiarea power system problems, many studies ignore the attacks from outside the system. There are many types of cyber-attacks that can act on the power systems, among them include denial-of-service attack, deception attack, and data integrity attack [17-21]. A deception attack is to compromise the trustworthiness of data by manipulating the packets transmitted over the communication networks. When under a deception attack, the flow of information in the system will be changed $[22,23]$, which may destabilize or even crash the system. We pay special attention to the deception attack in this study. In [24], a new malicious data deception attack by considering a practical attacking situation was investigated. An active synchronous detection method was proposed in [25] to detect deception attack in microgrids without impeding system operations. In [26], the authors investigated the viability of 
machine learning methods in detecting cyber-attacks. We want to get the information that is already damaged, and the observer-dependent event-triggered scheme is used in this study.

The event-trigger control works only when a certain set of event-trigger conditions are met. Using this method can not only make effective use of communication resources but also prolong the service time of the controller. This makes it be rapidly developed in the system control, especially in dealing with complex networked dynamic systems. A properly designed event-triggering scheme may also help us find out whether the system is under malicious attack. In [27], a dynamic event-triggered mechanism was proposed to modulate the transmission of data packets. The cooperative control problem has been investigated based on a novel event-triggered scheme in [28]. The random deception attack is well handled according to a memory-based event-triggered scheme proposed in [29]. In [30], a mathematical model concerning the event-triggered load frequency control under deception attack was developed. The collaborative design of both the eventtrigger strategy and the adaptive robust controller is investigated in [31]. The authors in [32] investigated the observerbased predictive event-triggered load frequency control for a smart grid incorporating electric vehicles. Benefiting from the use of an event-triggered mechanism, the negative impacts of deception attack on information transmission can be effectively detected, based on which the control module may work in time to ensure the stable operation of the system.

We design an observer-based sliding mode LFC scheme for controlling multiarea power systems under deception attack. First, by taking into account the characteristic of the deception attack, a model of the attacked power system is built. Then, an observer is proposed to estimate the state of the damaged system. Furthermore, the event-trigger is introduced to judge whether the normal operation of the system is compromised using the designed event-trigger conditions. The main contributions of this study can be generalized as follows:

(1) A novel sliding mode control was proposed for the multiarea power systems under deception attack. The proposed observe-based event-trigger sliding mode control helps save the limited network channel resources, so that the desired control can be performed successfully.

(2) The sliding mode load frequency problem in an interconnected power system with a deception attack was well-analyzed. Considering the designed sliding mode control surface, the equivalent control under the basic sliding mode control is obtained through analysis. The observer and the event-triggering scheme are introduced to analyze the power system under deception attack. Using the linear matrix inequality (LMI) method, the conditions of the system's asymptotic stability are obtained. Finally, an appropriate sliding mode control law is designed to ensure the reachability of the system trajectory.

The rest of this study is organized as follows. Section 2 introduces the modeling for the multiarea power system. The switching surface designed for the $i^{\text {th }}$ area of the power system is given in Section 3. Then, based on the designed sliding mode controller, the $H_{\infty}$ problem of the power system, and control law is developed to ensure the dynamic quality of sliding mode surface. Some illustrative examples of a three-area interconnected power system are analyzed to demonstrate the feasibility of the proposed approach in Section 4. Finally, Section 5 concludes our discussion.

\section{Problem Formulation}

2.1. Model Description. The multiarea power system is a type of the complex control system. In this study, the control problem of a sliding mode load frequency control-based power system under deception attack is analyzed. The $i^{\text {th }}$ area of a power system under the deception attack model is shown in Figure 1, which consists of a generator, a governor, a turbine, and so on. The meanings of the notations in Figure 1 are given in Table 1.

Based on the system model and information transfer, the dynamic equation of the system can be obtained as follows:

$$
\left\{\begin{array}{l}
\Delta \dot{P}_{m i}(t)=-\frac{\Delta P_{m i}(t)}{T_{\text {chi }}}+\frac{\Delta P_{v i}(t)}{T_{\text {chi }}}, \\
\Delta \dot{P}_{e i}(t)=\Delta P_{\mathrm{tie}}^{i}(t)+\beta_{i} \Delta f_{i}(t), \\
\Delta \dot{P}_{\mathrm{tie}}^{i}(t)=-2 \pi \sum_{j=1, i \neq j}^{n} T_{i j}\left(\Delta f_{i}(t)-\Delta f_{j}(t)\right), \\
\Delta \dot{P}_{v i}(t)=\frac{u_{i}(t)}{T_{g i}}-\frac{\Delta f_{i}(t)}{R_{i} T_{g i}}-\frac{\Delta P_{v i}(t)}{T_{g i}}-\frac{\Delta P_{e i}(t)}{T_{g i}}, \\
\Delta \dot{f}_{i}(t)=\frac{\Delta P_{m i}(t)}{M_{i}}-\frac{\Delta P_{d i}(t)}{M_{i}}-\frac{D_{i} \Delta f_{i}(t)}{M_{i}}-\frac{\Delta P_{\mathrm{tie}}^{i}(t)}{M_{i}} .
\end{array}\right.
$$

We can derive the state equation of $i^{\text {th }}$ area power system as

$$
\left\{\begin{array}{l}
\dot{x}(t)=A x(t)+B u(t)+F w(t), \\
y(t)=C x(t),
\end{array}\right.
$$

where $w(t)$ is the disturbance of the system and defined as $w(t)=\Delta P_{d i}$, and $w(t)$ is bounded and satisfies $\Delta P_{d i}<h_{i}$, where $h_{i}$ represent the positive constants,

$$
\begin{aligned}
x_{i}(t) & =\left[x_{1}^{T}(t), x_{2}^{T}(t), \ldots, x_{n}^{T}(t)\right]^{T}, \\
u(t) & =\left[u_{1}^{T}(t), u_{2}^{T}(t), \ldots, u_{n}^{T}(t)\right]^{T}, \\
y_{i}(t) & =\left[y_{1}^{T}(t), y_{2}^{T}(t), \ldots, y_{n}^{T}(t)\right]^{T}, \\
w_{i}(t) & =\left[\Delta P_{d 1}(t), \Delta P_{d 2}(t), \ldots, \Delta P_{d n}(t)\right]^{T}, \\
x(t) & =\left[\Delta f_{i}, \Delta P_{m i}, \Delta P_{v i}, \Delta P_{e i}, \Delta P_{\mathrm{tie}}^{i}\right]^{T},
\end{aligned}
$$

where $A, B, C$, and $F$ are the parameter matrixes,

$$
\begin{aligned}
& A=\left[A_{i j}\right]_{n \times n}, \quad B=\operatorname{diag}\{\underbrace{B_{1}, \ldots, B_{i}}_{n}\}, \quad C=\operatorname{diag} \\
& \{\underbrace{C_{1}, \ldots, C_{i}}_{n}\}, \text { and } F=\operatorname{diag}\{\underbrace{F_{1}, \ldots, F_{i}}_{n}\} .
\end{aligned}
$$




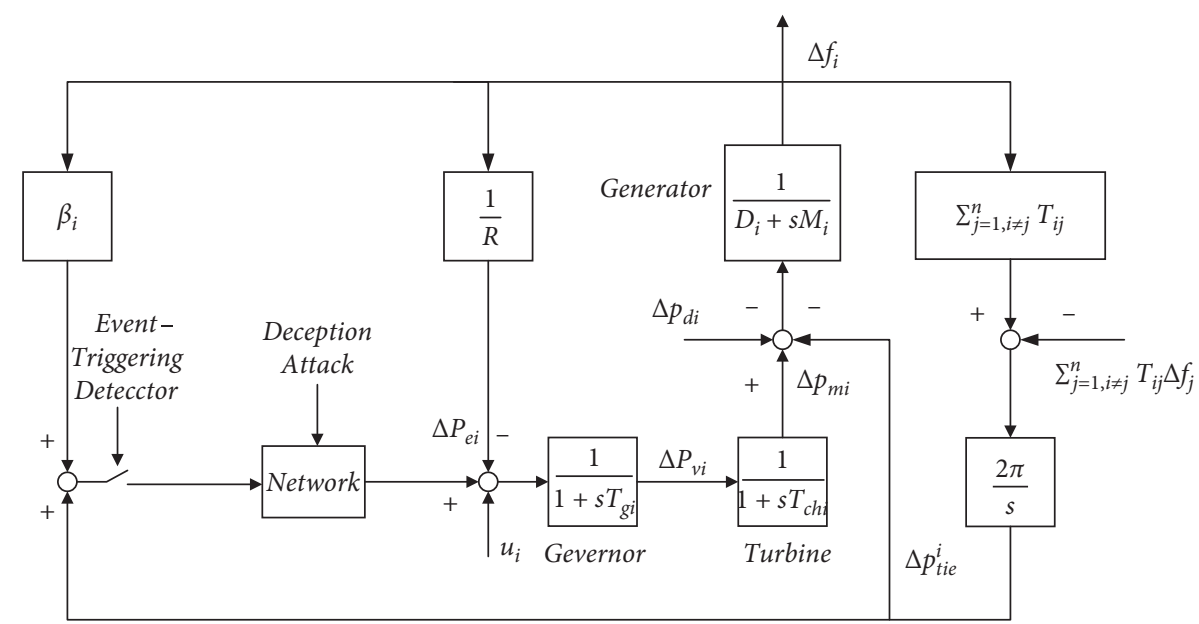

Figure 1: The model of the $i^{\text {th }}$ area of the power system.

TABLE 1: Definition of notations.

\begin{tabular}{lc}
\hline$\Delta f_{i}$ & Frequency deviation \\
\hline$\Delta P_{\text {tie }}^{i}$ & Deviation of tie-line power exchange between areas \\
$\Delta P_{m i}$ & Mechanical output deviation \\
$\Delta P_{d i}$ & Load deviation \\
$D_{i}$ & Damping coefficient \\
$M_{i}$ & Inertia of the generator \\
$\Delta P_{v i}$ & The deviation value \\
$R_{i}$ & Droop coefficient \\
$T_{g i}$ & Time constant of the governor \\
$u_{i}$ & Control input \\
$T_{\text {chi }}$ & Time constant of the turbine \\
$T_{i j}$ & Synchronization coefficient between areas \\
\hline
\end{tabular}

$$
\begin{aligned}
& A=\left[\begin{array}{ccccc}
-\frac{D_{i}}{M_{i}} & \frac{1}{M_{i}} & 0 & 0 & -\frac{1}{M_{i}} \\
0 & -\frac{1}{T_{c h i}} & \frac{1}{T_{c h i}} & 0 & 0 \\
-\frac{1}{R_{i} T_{g i}} & 0 & -\frac{1}{T_{g i}} & 0 & 0 \\
\beta_{i} & 0 & 0 & 0 & 1 \\
-2 \pi \sum_{j=1, j \neq i}^{n} T_{i j} & 0 & 0 & 0 & 0
\end{array}\right] \text {, } \\
& B=\left[\begin{array}{lllll}
0 & 0 & \frac{1}{T_{g i}} & 0 & 0
\end{array}\right]^{T}, \\
& F=\left[\begin{array}{lllll}
0 & 0 & 0 & 0 & -\frac{1}{M_{i}}
\end{array}\right]^{T} \text {. }
\end{aligned}
$$

2.2. Event-Triggered Control. The data information of the power system is transmitted by the shared network to the corresponding sliding mode controller. In this section, a sliding mode observer-based load frequency controller for the $i^{\text {th }}$ area power systems is given. An event-triggered scheme will be put forward taking into account the consideration of the deception attack:

$$
\tilde{y}(t)=(1-\alpha(t)) y(t)+\alpha(t) \varepsilon(t),
$$

where $\varepsilon(t)$ is the deception attack from outside of the system and $\alpha(t)$ is an energy-bounded signal belonging to $L_{2} \in[0,+\infty) . \alpha(t)$ is a stochastic variable subject to a Bernoulli distribution satisfying

$$
\left\{\begin{array}{l}
P\{\alpha(t)=1\}=\mathbb{E}\{\alpha(t)\}:=\alpha_{0}, \\
P\{\alpha(t)=0\}=1-\mathbb{E}\{\alpha(t)\}:=1-\alpha_{0}
\end{array}\right.
$$

When the deception attack happens, the information transmitted will be changed, and the system equation of state can be expressed as

$$
\left\{\begin{array}{l}
\dot{x}(t)=A x(t)+B u(t)+F w(t), \\
\tilde{y}(t)=(1-\alpha(t)) C x(t)+\alpha(t) \mathcal{E}(t) .
\end{array}\right.
$$

Using the observed information as the transmit information, to facilitate the analysis of the problem, the state of the observed information is defined as $\widehat{x}(t)$; then, 


$$
\left\{\begin{array}{l}
\dot{\hat{x}}(t)=A \widehat{x}(t)+B u(t)+L(\tilde{y}(t)-\hat{y}(t)) \\
\hat{y}(t)=(1-\alpha(t)) C \hat{x}(t)
\end{array}\right.
$$

To facilitate the detection of the deception attack on the system, the event-trigger is used in the system model (1). In the absence of deception attack, a common event-triggered condition [33] is given as

$$
t_{k+1} h=t_{k} h+\min _{d \in \mathbb{R}^{+}}\left\{d h \| e^{T}\left(j_{k} h\right) \phi e\left(j_{k} h\right) \geq \delta\left(t_{k} h\right) \hat{x}^{T}\left(t_{k} h\right) \phi \widehat{x}\left(t_{k} h\right)\right\},
$$

where $\phi=\eta C^{T} C, \eta$ is a positive definite matrix, and $\phi=$ $\operatorname{diag}\left\{\phi_{1}, \ldots, \phi_{n}\right\}$ is defined as a weighting matrix. We denote the information error as $e\left(j_{k} h\right)=\hat{x}\left(j_{k} h\right)-\hat{x}\left(t_{k} h\right)$, $j_{k} h=t_{k} h+d h, d \in \mathbb{R}_{0}^{+} . \delta$ is a predefined scalar confined in the interval $[0,1)$.

The system error is $\Theta(t)=x(t)-\widehat{x}(t)$. Then,

$$
\dot{\Theta}(t)=A \Theta(t)+F w(t)-L(\alpha(t) \varepsilon(t)+(1-\alpha(t)) C \Theta(t)) .
$$

2.3. Sliding Mode Design. To make the system working stable, the sliding mode control method is introduced. We construct the following sliding mode surface function:

$$
\sigma(t)=G \widehat{x}(t)-\int_{0}^{t} G(A+B K) \widehat{x}(\tau) \mathrm{d} \tau,
$$

where $G$ is a constant matrix, and it is selected to ensure that $G B$ stays nonsingular, $0 \leq \tau(t) \leq h+\tau_{\max }, \tau_{\max }>0$.

We all know that the switch surface function should satisfy

$$
\begin{aligned}
\sigma(t) & =0, \\
t \dot{\sigma}(t) & =0 .
\end{aligned}
$$

The derivative of the sliding surface is

$$
\dot{\sigma}(t)=G \dot{\hat{x}}(t)-G A \hat{x}(t)-G B K \widehat{x}(t) .
$$

According to (8) and (13), we have

$$
\begin{aligned}
\dot{\sigma}(t)= & G[A \hat{x}(t)+B u(t)+L(\tilde{y}(t)-\hat{y}(t))] \\
& -G A \hat{x}(t)-G B K \hat{x}(t) \\
= & -G B K \hat{x}(t)+G B u(t)+G L(\widetilde{y}(t)-\widehat{y}(t)) .
\end{aligned}
$$

Noticing the function (12), we have

$$
-G B K \hat{x}(t)+G B u(t)+G L(\tilde{y}(t)-\hat{y}(t))=0 .
$$

Thus, we get the equivalent controller:

$$
u_{\mathrm{eq}}=K \hat{x}(t)-(G B)^{-1} G L(\tilde{y}(t)-\hat{y}(t)) .
$$

Then, the controlled system and the error system are reformed as

$$
\left\{\dot{\hat{x}}(t)=(A+B K) \hat{x}(t)+\left(L-B(G B)^{-1} G L\right)(\alpha(t) \varepsilon(t)+(1-\alpha(t)) C \Theta(t)), \dot{\Theta}(t)=A \Theta(t)+F w(t)-L(\alpha(t) \varepsilon(t)+(1-\alpha(t)) C \Theta(t)) .\right.
$$

\section{Main Results}

In this section, sufficient conditions are established for the closed-loop system (15) to be globally asymptotically stable with an L-O norm bound. Before presenting the main results, the following useful lemma is introduced.

Definition 1. Considering the power system (14), if $w(t)=0$ and $\varepsilon(t)=0$, there exist a positive definite continuous firstorder partial derivative $V(\widehat{x}(t))$ and negative definite $\mathbb{E}\{\dot{V}(\hat{x}(t))\}$. One can conclude that the system is asymptotically stable. Besides, under the zero initial condition of $\widehat{x}(t)=0, t \in[-\tau, 0]$, any nonzero $w(t), \varepsilon(t) \in L_{2}[0,+\infty)$. The disturbance rejection level of $H_{\infty}$ is given as $\gamma$, and we have the following condition: $\mathbb{E}\left\{\|\hat{y}(t)\|_{2}\right\} \leq \gamma$ $\mathbb{E}\left\{\|w(t)\|_{2}+\|\varepsilon(t)\|_{2}\right\}$.
Lemma 1. For any real vectors $u$ and $v$ and symmetric positive matrix $Q$ with compatible dimensions, the following inequality holds:

$$
u^{T} v+v^{T} u \leq u^{T} Q u+v^{T} Q^{-1} v .
$$

\subsection{Stability Analysis}

Theorem 1. For the given conditions $1>\alpha_{0} \geq 0$, system (5) is asymptotically stable for the given positive constants $\gamma$ and real symmetric matrices $Q>0, W>0$ satisfying the following matrix inequality:

$$
\left[\begin{array}{ll}
\omega_{11} & \omega_{12} \\
\omega_{12}^{T} & \omega_{22}
\end{array}\right]<0
$$


where

$$
\begin{aligned}
& \omega_{11}=\omega_{a}-\omega_{b}, \\
& \omega_{a}=\left[a_{7 * 7}\right] \text {, } \\
& \omega_{b}=\Gamma^{T} \Gamma \text {, } \\
& a_{11}=\operatorname{sym}[X(A+B K)]+Q \text {, } \\
& a_{13}=\left(1-\alpha_{0}\right) X L C \text {, } \\
& a_{17}=\alpha_{0} X L \text {, } \\
& a_{22}=\delta\left(j_{k} h\right) \phi \text {, } \\
& a_{25}=-\delta\left(j_{k} h\right) \phi \text {, } \\
& a_{33}=\operatorname{sym}[X A]+W-\left(1-\alpha_{0}\right) X L C \text {, } \\
& a_{36}=X F \text {, } \\
& a_{37}=-\alpha_{0} X L \text {, } \\
& a_{55}=\left(\delta\left(j_{k} h\right)-1\right) \phi \text {, } \\
& a_{66}=a_{77}=-\gamma^{2} I \text {, } \\
& \Gamma=\left[\begin{array}{ll}
\left(1-\alpha_{0}\right) C & 0^{1 * 6}
\end{array}\right] \text {, } \\
& \varpi_{12}=\left[\begin{array}{ccc}
X B & 0 & 0 \\
0 & 0 & 0 \\
0 & \left(1-\alpha_{0}\right) C^{T} L^{T} X & 0 \\
0^{3 \times 1} & 0^{3 \times 1} & 0^{3 \times 1} \\
0 & 0 & \alpha_{0} L^{T} X
\end{array}\right] \text {, } \\
& \omega_{22}=\left[\begin{array}{ccc}
-G B & 0 & 0 \\
0 & -\left(1-\alpha_{0}\right) X & 0 \\
0 & 0 & -\alpha_{0} X
\end{array}\right] \text {. }
\end{aligned}
$$

Proof. Propose a Lyapunov functional

$$
V(t)=V_{\widehat{x}}(t)+V_{\Theta}(t),
$$

where

$$
\left\{\begin{array}{l}
V_{\widehat{x}}(t)=\widehat{x}^{T}(t) X \widehat{x}(t)+\int_{t-\tau(t)}^{t} \widehat{x}^{T}(v) Q \widehat{x}(v) \mathrm{d} v, \\
V_{\Theta}(t)=\Theta^{T}(t) X \Theta(t)+\int_{t-\tau(t)}^{t} \Theta^{T}(v) W \Theta(v) \mathrm{d} v .
\end{array}\right.
$$

$$
\begin{aligned}
\dot{V}(t) & =\dot{V}_{\widehat{x}}(t)+\dot{V}_{\Theta}(t), \\
& \left\{\begin{array}{l}
\dot{V}_{\widehat{x}}(t)=2 \widehat{x}^{T}(t) X \dot{\hat{x}}(t)+\widehat{x}^{T}(t) Q \widehat{x}(t), \\
\dot{V}_{\Theta}(t)=2 \Theta^{T}(t) X \Theta(t)+\Theta^{T}(t) W \Theta(t) .
\end{array}\right.
\end{aligned}
$$

The expectation of (23) is

$$
E\{\dot{V}(t)\}=E\left\{\dot{V}_{\widehat{x}}(t)+\dot{V}_{\Theta}(t)\right\},
$$

$$
\begin{aligned}
E\left\{\dot{V}_{\widehat{x}}(t)\right\}= & 2 \widehat{x}^{T}(t) X(A+B K) \widehat{x}(t)+2 \alpha_{0} \widehat{x}^{T}(t) X\left(L-B(G B)^{-1} G L\right) \varepsilon(t) \\
& +2\left(1-\alpha_{0}\right) \widehat{x}^{T}(t) X\left(L-B(G B)^{-1} G L\right) C \Theta(t)+2 \Theta^{T}(t) X F \omega(t)+\widehat{x}^{T}(t) Q \widehat{x}(t), \\
E\left\{\dot{V}_{\Theta}(t)\right\}= & 2 \Theta^{T}(t) X A \Theta(t)-2 \alpha_{0} \Theta^{T}(t) X L \varepsilon(t) \\
& -2\left(1-\alpha_{0}\right) \Theta^{T}(t) X L C \Theta(t)+\Theta^{T}(t) W \Theta(t)+e^{T}\left(j_{k} h\right) \phi e\left(j_{k} h\right)-e^{T}\left(j_{k} h\right) \phi e\left(j_{k} h\right) .
\end{aligned}
$$


Notice that there exist some complex items in (24). According to Lemma 1, these complex items can be handled like

$$
-2 \alpha_{0} \widehat{x}^{T}(t) X B(G B)^{-1} G L \varepsilon(t) \leq \alpha_{0} \widehat{x}^{T}(t) X B(G B)^{-1} G \widehat{x}(t)+\alpha_{0} \varepsilon^{T}(t) L^{T} X L \varepsilon(t) .
$$

Also, we have

$$
-2\left(1-\alpha_{0}\right) \hat{x}^{T}(t) X B(G B)^{-1} G L C \Theta(t) \leq\left(1-\alpha_{0}\right) \hat{x}^{T}(t) X B(G B)^{-1} G \widehat{x}(t)+\left(1-\alpha_{0}\right) \times \Theta^{T}(t) C^{T} L^{T} X L C \Theta(t) .
$$

Take the event-triggered condition into considering

$$
e^{T}\left(j_{k} h\right) \phi e\left(j_{k} h\right)<\delta\left(j_{k} h\right)\left(\widehat{x}(t-\tau(t))-e\left(j_{k} h\right)\right)^{T} \phi\left(\widehat{x}(t-\tau(t))-e\left(j_{k} h\right)\right) .
$$

For convenience, we define a matrix as follows:

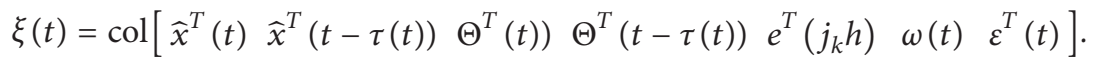

Then,

$$
\begin{aligned}
& \mathbb{E}\{V(t)\}+\mathbb{E}\left\{\widehat{y}^{T}(t) \hat{y}(t)\right\}-\gamma^{2} \mathbb{E}\left\{\omega^{T}(t) \omega(t)+\varepsilon^{T}(t) \varepsilon(t)\right\} \\
& \leq \xi^{T}(t)\left(\varpi_{11}-\varpi_{12} \Phi_{22}^{-1} \varpi_{12}^{T}\right) \xi(t) .
\end{aligned}
$$
as

Using the Schur theorem given in [34], (30) is concluded

$$
\mathbb{E}\{V(t)\} \leq \gamma^{2} \mathbb{E}\left\{\omega^{T}(t) \omega(t)-\widehat{y}^{T}(t) \hat{y}(t)+\varepsilon^{T}(t) \varepsilon(t)\right\} .
$$

Integrating the deduction above, we have

$$
\mathbb{E}\{V(+\infty)\}-\mathbb{E}\{V(0)\} \leq \mathbb{E}\left\{\int_{0}^{+\infty}\left[-\widehat{y}^{T}(t) \widehat{y}(t)+\gamma^{2}\left(\omega^{T}(t) \omega(t)+\varepsilon^{T}(t) \varepsilon(t)\right)\right]\right\} \mathrm{d} t
$$

Under zero initial condition, we can further obtain

$$
\mathbb{E}\left\{\|\hat{y}(t)\|_{2}\right\} \leq \gamma \mathbb{E}\left\{\|\omega(t)\|_{2}\right\}+\mathbb{E}\left\{\|\varepsilon(t)\|_{2}\right\} .
$$

When $\omega(t)=0$ and $\varepsilon(t)=0$, the inequality is concluded as

$$
\mathbb{E}\{\dot{V}(t)\} \leq-\mathbb{E}\left\{\hat{y}^{T}(t) \hat{y}(t)\right\}<0 .
$$

Then, there exists a positive scalar $\epsilon>0$ that makes the following inequality holds

$$
\mathbb{E}\{\dot{V}(t)\} \leq-\epsilon \mathbb{E}\left\{\|\widehat{x}(t)\|^{2}\right\}
$$

Based on the proposed control scheme, the multiarea power system is to be robustly stable with an optimal attenuation level $\gamma$. It completes the proof.

Corollary 1. System (5) is asymptotically stable for the given conditions $\alpha_{0} \geq 0$, if there exist matrixes $Q>0, W>0$, appropriate known positive number $\gamma>0$, real symmetric matrixes $\chi>0$, and an arbitrary matrix $Y$ satisfying the following LMI:

$$
\left[\begin{array}{ccc}
\widetilde{\varpi}_{11} & \widetilde{\varpi}_{12} & \widetilde{\varpi}_{13} \\
* & \widetilde{\varpi}_{22} & 0^{1 * 3} \\
* & * & -I
\end{array}\right]<0,
$$


where

$$
\begin{aligned}
& \widetilde{\mathrm{\omega}}_{11}=\left[\widetilde{a}_{7 * 7}\right], \\
& \tilde{a}_{11}=\operatorname{sym}[A \chi+B Y]+\chi Q \chi \text {, } \\
& \tilde{a}_{13}=\left(1-\alpha_{0}\right) L C \chi \text {, } \\
& \tilde{a}_{17}=\alpha_{0} L \text {, } \\
& \tilde{a}_{22}=\delta\left(j_{k} h\right) \phi \text {, } \\
& \tilde{a}_{25}=-\delta\left(j_{k} h\right) \phi \text {, } \\
& \tilde{a}_{33}=\operatorname{sym}[A \chi]+\chi W \chi-\left(1-\alpha_{0}\right) L C \chi, \\
& \tilde{a}_{36}=F \text {, } \\
& \tilde{a}_{37}=-\alpha_{0} L \text {, } \\
& \tilde{a}_{55}=\left(\delta\left(j_{k} h\right)-1\right) \phi, \\
& \tilde{a}_{66}=\tilde{a}_{77}=-\gamma^{2} I, \\
& \widetilde{\mathrm{\omega}}_{13}=\left[\begin{array}{ll}
\left(1-\alpha_{0}\right) C \chi & 0^{1 * 6}
\end{array}\right]^{T} \text {, } \\
& \widetilde{\varpi}_{12}=\left[\begin{array}{ccc}
B & 0 & 0 \\
0 & 0 & 0 \\
0 & \left(1-\alpha_{0}\right) \chi^{T} C^{T} N^{T} & 0 \\
0^{3 \times 1} & 0^{3 \times 1} & 0^{3 \times 1} \\
0 & 0 & \alpha_{0} L^{T}
\end{array}\right] \text {, } \\
& \widetilde{\omega}_{22}=\left[\begin{array}{ccc}
-G B & 0 & 0 \\
0 & -\left(1-\alpha_{0}\right) \chi & 0 \\
0 & 0 & -\alpha_{0} \chi
\end{array}\right] \text {. }
\end{aligned}
$$

Proof. Define $\chi=X^{-1}$ and $Y=K \chi$, pre and postmultiplying both sides of inequality (16) with $\operatorname{diag}\{\chi, I, \chi, I, I, I, I, I, \chi, \chi\}$.

We can notice that the matrix in Theorem 2 cannot be solved directly. The reason is the nonlinear terms $L C \chi, \chi W \chi$, and $\chi Q \chi$. To deal with the nonlinear term $L C \chi$ in Theorem 2, we have added the following theorem, so that the nonlinear problem is solvable. Besides, $\chi W \chi$ and $\chi Q \chi$ are defined as $\chi W \chi=\widetilde{W}$ and $\chi Q \chi=\widetilde{Q}$, respectively.

Theorem 2. Based on Corollary 1, for the given full rank matrix $M$ and any proper matrix $N$ with appropriate dimensions, (24) holds, and the following LMI:

$$
\begin{gathered}
{\left[\begin{array}{ccc}
\widetilde{\Phi}_{11}^{\prime} & \widetilde{\Phi}_{12}^{\prime} & \widetilde{\Phi}_{13} \\
* & \widetilde{\Phi}_{22} & 0^{1 * 3} \\
* & * & -I
\end{array}\right]<0,} \\
{\left[\begin{array}{ccc}
\xi I & C^{T} M^{T}-X^{T} C^{T} \\
* & & -I
\end{array}\right]<0,}
\end{gathered}
$$

where $\xi$ is approaching to $0, \widetilde{\Phi}_{11}^{\prime}$ and $\widetilde{\Phi}_{12}^{\prime}$ are obtained from $\widetilde{\Phi}_{11}$ and $\widetilde{\Phi}_{12}$ by replacing $L C \chi$ with $N C$ and replacing $\chi W \chi$ and $\chi Q \chi$ with $\widetilde{W}$ and $\widetilde{Q}$ respectively, and the system is asymptotically stable with observe gains $L=N M^{-1}$. It completes the proof.

Remark 1. Based on the proposed sliding mode load frequency control scheme, Theorem 2 could guarantee the attacked multiarea power system to be robustly stable with an optimal attenuation level $\gamma$. In Theorem 2, the nonlinear coupling terms has been replaced by processable terms.

3.2. Control Law Design. For sliding mode control theory, a proper sliding control law is of great importance. The trajectory of the power system shall be reachable to the sliding surface in a limited time under the supplied controller. For the controlled multiarea power system (7), the corresponding reachability condition for the $i^{\text {th }}$ area can be described by the following theorem.

Theorem 3. For the considered sliding mode surface (9), a proper control law is given as

$$
\begin{aligned}
u(t)= & K \hat{x}(t)-(1-\alpha(t))(G B)^{-1} G L C \Theta(t)-h_{i}(G B)^{-1} \sigma_{i}(t) \\
& -h_{i}(G B)^{-1}\|G F\| \operatorname{sgn}\left(\sigma_{i}(t)\right),
\end{aligned}
$$

where $h_{i}$ is a positive constant, and $\operatorname{sgn}\left(\sigma_{i}(t)\right)$ is the switching function:

$$
\operatorname{sgn}\left(\sigma_{i}(t)\right)= \begin{cases}-1, & \text { if } \sigma_{i}(t)<0, \\ 0, & \text { if } \sigma_{i}(t)=0, \\ 1, & \text { if } \sigma_{i}(t)>0 .\end{cases}
$$

Proof. The Lyapunov function is constructed as

$$
V_{L}=\frac{1}{2} \sigma_{i}^{T}(t) \sigma_{i}(t) .
$$

From (14), (39), and (41), the derivative of $V_{L}(t)$ is 


$$
\begin{aligned}
\dot{V}_{L}= & \frac{1}{2} \dot{\sigma}_{i}^{T}(t) \sigma_{i}(t)+\frac{1}{2} \sigma_{i}^{T}(t) \dot{\sigma}_{i}(t) \\
= & \sigma_{i}^{T}(t) \dot{\sigma}_{i}(t) \\
= & \sigma_{i}(t)[-G B K \hat{x}+G B u(t)+(1-\alpha(t)) G L C \Theta(t)] \\
= & \sigma_{i}(t)\left[-G B K \hat{x}+(1-\alpha(t)) G L C \Theta(t)+G B\left(K \hat{x}(t)-(1-\alpha(t))(G B)^{-1} G L C \Theta(t)\right.\right. \\
& \left.\left.-h_{i}(G B)^{-1} \sigma_{i}(t)-h_{i}(G B)^{-1}\|G F\| \operatorname{sgn}\left(\sigma_{i}(t)\right)\right)\right] \\
= & -h_{i}\left\|\sigma_{i}(t)\right\|^{2}-h_{i} \sigma_{i}(t)\|G F\| \operatorname{sgn}\left(\sigma_{i}(t)\right) \\
& <-h_{i}\left\|\sigma_{i}(t)\right\|^{2}-h_{i}\left\|\sigma_{i}(t)\right\|\|G F\| \\
& <0 .
\end{aligned}
$$

Therefore, one can conclude that the system can be kept stable by the designed control law (39).

Remark 2. The variable structure control has the advantages of fast response, being insensitive to parameter changes and disturbances. The way of sliding mode control can be designed and independent of plant parameters and disturbances. This method can be simple implemented in physical. The main disadvantage, however, is that the imperfect of all kinds of components of the actual system may contribute to causing the control switching delay. Specifically, when the state trajectory reaches the sliding surface, it is difficult to slide strictly along the sliding surface towards the equilibrium point. Instead, it may pass back and forth on both sides of the sliding surface, resulting in chattering, the error of the operation in MATLAB. Besides, the use of sliding mode control will certainly cause some buffeting phenomena.

\section{Numerical Examples}

In this section, numerical examples are provided to demonstrate the effectiveness of the proposed design scheme. Taking a three-area interconnected power system as an example, the simulation results verify the effectiveness and correctness of the proposed sliding mode control scheme. By analyzing the simulation diagram, one can conclude that the system can reach a stable state under the designed sliding mode control.

Parameter values of the three-area interconnected power system are presented in Table 2 . We consider a three-area interconnected power system. By using the MATLAB LMI control toolbox, the stability condition could be verified effectively.
The controller gain and observe gain can be calculated as

$$
\begin{aligned}
K & =\left[\begin{array}{llllll}
-223.783 & -5.012 & -3.280 & 91.864 & 1.795 \\
-157.956 & -2.073 & -2.597 & 67.533 & 1.631 \\
-160.963 & -2.360 & -2.448 & 69.519 & 1.510
\end{array}\right], \\
L & =\left[\begin{array}{llllll}
0.272 & 1.219 & -0.280 & 0.075 & -0.116 \\
0.108 & -0.04 & -0.224 & 0.019 & -0.295 \\
0.207 & -0.017 & -0.292 & 0.063 & -0.135
\end{array}\right]^{T}
\end{aligned}
$$

Under the control scheme, the frequency derivative of the power system is shown in Figure 2. It can be seen that with the designed control scheme, the frequency derivative value of the deception attacked power system gradually tends to a stable state. This demonstrates that the proposed controller can effectively reduce the frequency deviation of the system, improving the robustness of the attacked power system, that is, the power system can be stable under sliding mode control, although it was attacked.

The designed sliding surface trajectory is shown in Figure 3. It can be seen from the figure that the sliding surface can reach a stable state in a certain period and keep running. One can prove that the sliding mode which we designed can work normally and satisfies our purpose.

It can be seen from Figure 4 that the power system under equivalent control can operate to a stable state over time. This means the power system can work in a stable state under the provided control method.

To ensure that the system can reach the sliding surface in a limited time, the state trajectory of the sliding mode control law is shown in Figure 5. According to the curve, the 
TABle 2: Parameters of the three-area interconnected power system.

\begin{tabular}{lc}
\hline$D_{1}, D_{2}, D_{3}$ & $1,1.5,1.8$ \\
\hline$T_{c h 1}, T_{c h 2}, T_{c h 3}$ & $0.3,0.17,0.2$ \\
$T_{g 1}, T_{g 2}, T_{g 3}$ & $0.37,0.4,0.35$ \\
$R_{1}, R_{2}, R_{3}$ & $0.05,0.05,0.05$ \\
Beta 1, beta 2, beta 3 & $21,21.5,21.8$ \\
$P_{d 1}, P_{d 2}, P_{d} 3$ & $0.1,0.1,0.1$ \\
$\gamma_{1}, \gamma_{2}, \gamma_{3}$, & $2,2,2$ \\
\hline
\end{tabular}

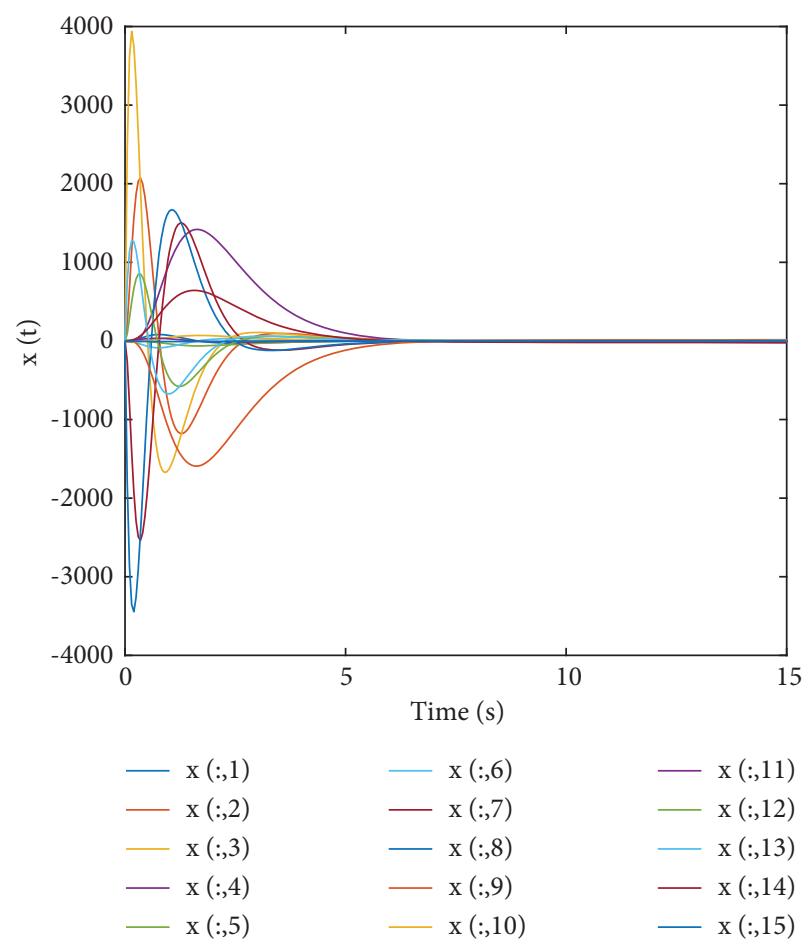

FIgURE 2: Frequency deviation of the power system.

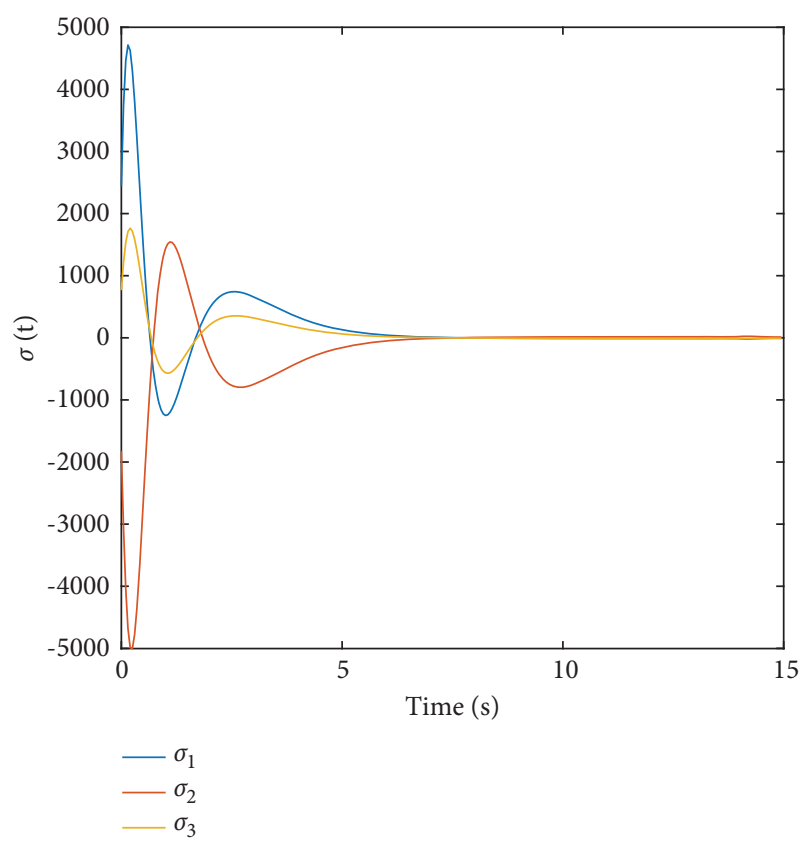

Figure 3: Designed sliding mode surface.

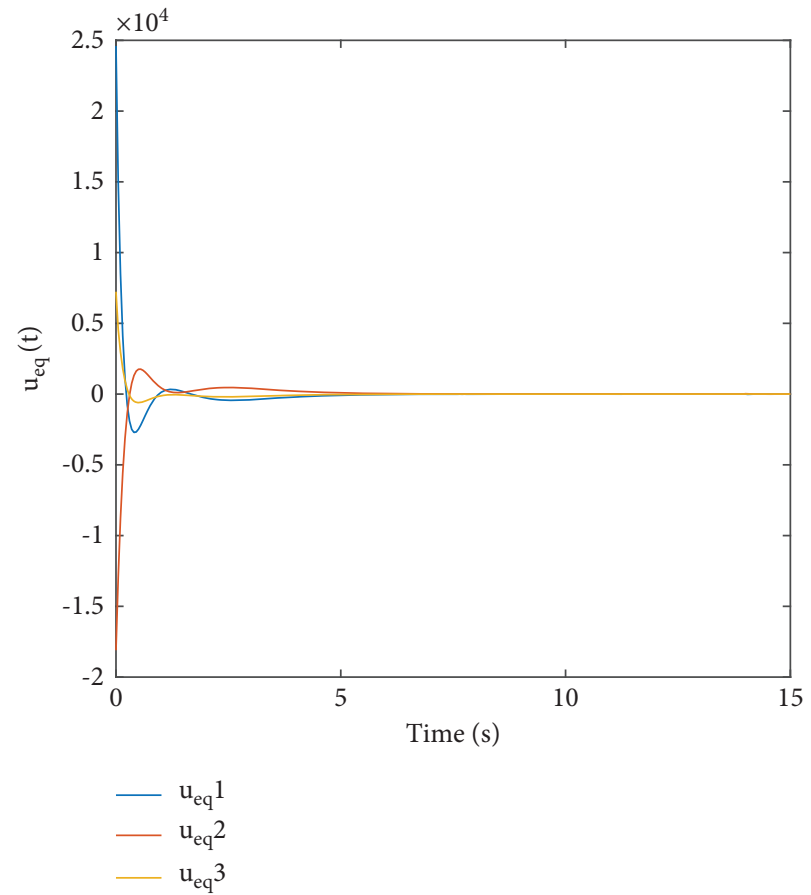

FIGURE 4: Equivalent control of the sliding mode-controlled power system.

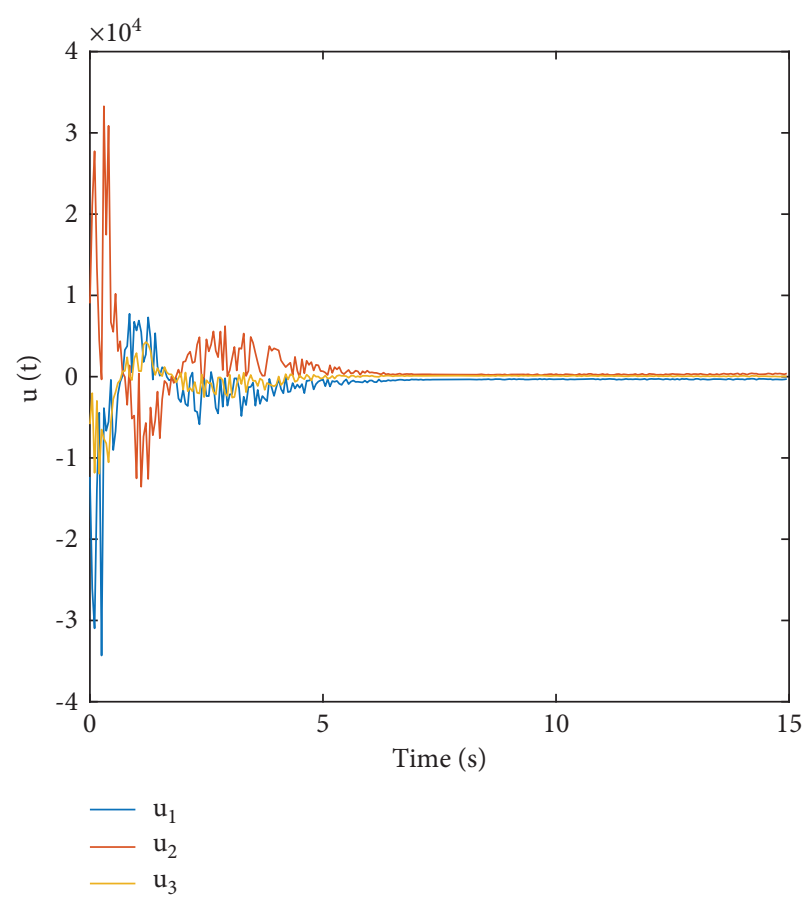

FIgURE 5: State of sliding mode control law.

simulation results can easily show the effectiveness of the proposed sliding mode load frequency control scheme.

We test on the case where something is random and the event-trigger frequency and probability are equally random. Figure 6 shows the event interval during the deception 


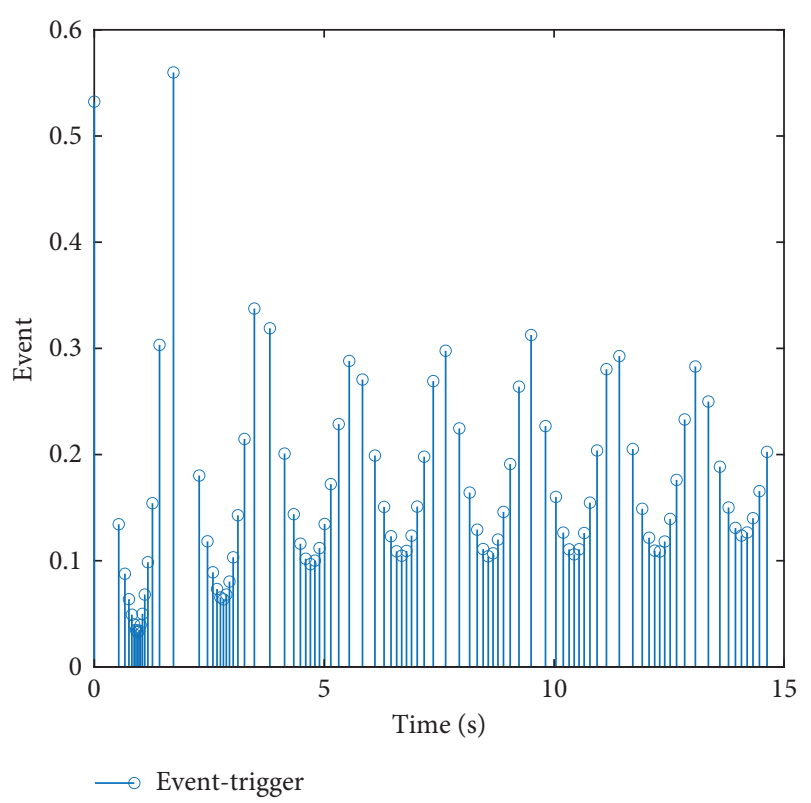

FIGURE 6: Event interval during deception attack interval.

attack. The disorder event is shown in Figure 6, conforming to our requirements for random attacks.

\section{Conclusion}

This study proposed a sliding mode load frequency control for multiarea power systems under deception attack. An observer-based event-triggered transmission scheme was proposed for multiarea power systems. To be specific, an observer has been provided to gain disturbed transmission information. An event-trigger scheme is added to judge the attack is occurred or not. On the basis of the above analysis, a sliding mode controller has been constructed to keep the stability of the multiarea power system which is injured by some attack. This study has considered a kind of attack. In fact, it is possible that several attacks influence the system at the same time. The modeling of attack is also a problem worthy of consideration. In addition, with more and more renewable energy access to the power grid, its inherent randomness and uncertainty should be paid attention to. It will be a research direction in the future to consider the random disturbance and distribution caused by renewable energy into the power system.

\section{Data Availability}

The data used to support the findings of this study were supplied by the Xi'an University of Technology under license and so cannot be made freely available. The data are available from the corresponding author Xinghua Liu upon request.

\section{Conflicts of Interest}

The authors declare that there are no conflicts of interest.

\section{Acknowledgments}

This work was partly supported by the National Natural Science Foundation of China (61903296 and U2003110), Innovative Talents Promotion Program Young Science and Technology Nova Project (2020KJXX-094), Key Laboratory Project of Shaanxi Educational Committee (20JS110), and High Level Plan of Shaanxi Province for Young Professionals.

\section{References}

[1] R. W. Kenyon, M. Bossart, M. Marković et al., "Stability and control of power systems with high penetrations of inverterbased resources: an accessible review of current knowledge and open questions," Solar Energy, vol. 210, no. 1, pp. 149-168, 2020.

[2] H. Ahsan and M. D. Mufti, "Comprehensive power system stability improvement with ROCOF controlled smes," Electric Power Components and Systems, vol. 48, no. 2, pp. 162-173, 2020.

[3] A. Xue, J. Zhang, L. Zhang, Y. Sun, J. Cui, and J. Wang, "Transient frequency stability emergency control for the power system interconnected with offshore wind power through VSC-HVDC," IEEE Access, vol. 8, pp. 53133-53140, 2020.

[4] X. Lv, Y. Sun, Y. Wang, and V. Dinavahi, "Adaptive eventtriggered load frequency control of multi-area power systems under networked environment via sliding mode control," IEEE Access, vol. 8, pp. 86585-86594, 2020.

[5] Y. M. Alsmadi, A. Alqahtani, R. Giral et al., "Sliding mode control of photovoltaic based power generation systems for microgrid applications," International Journal of Control, vol. 94, no. 6, pp. 1704-1715, 2021.

[6] M. Y. Silaa, M. Derbeli, O. Barambones, and A. Cheknane, "Design and implementation of high order sliding mode control for PEMFC power system," Energies, vol. 13, no. 17, pp. 4317-4332, 2020.

[7] A. T. Tran, B. Le Ngoc Minh, P. T. Tran et al., "Adaptive integral second-order sliding mode control design for load frequency control of large-scale power system with communication delays," Complexity, vol. 2021, Article ID 5564184, 2021.

[8] R. Nie, S. He, F. Liu, and X. Luan, "Sliding mode controller design for conic-type nonlinear semi-Markovian jumping systems of time-delayed chua's circuit," IEEE Transactions on Systems, Man, and Cybernetics: Systems, vol. 51, no. 4, pp. 2467-2475, 2021.

[9] G. Zhu, L. Nie, Z. Lv, L. Sun, X. Zhang, and C. Wang, "Adaptive fuzzy dynamic surface sliding mode control of large-scale power systems with prescribe output tracking performance," ISA Transactions, vol. 99, pp. 305-321, 2020.

[10] X. Lv, Y. Sun, W. Hu, and V. Dinavahi, "Robust load frequency control for networked power system with renewable energy via fractional-order global sliding mode control," IET Renewable Power Generation, vol. 15, no. 5, pp. 1046-1057, 2021.

[11] A.-T. Tran, B. L. N. Minh, V. V. Huynh et al., "Load frequency regulator in interconnected power system using second-order sliding mode control combined with state estimator," Energies, vol. 14, no. 4, pp. 863-880, 2021.

[12] M. Elsisi, N. Bazmohammadi, J. M. Guerrero, and M. A. Ebrahim, "Energy management of controllable loads in 
multi-area power systems with wind power penetration based on new supervisor fuzzy nonlinear sliding mode control," Energy, vol. 221, pp. 119867-119881, 2021.

[13] S. He, W. Lyu, and F. Liu, "Robust Ho sliding mode controller design of a class of time-delayed discrete conic-type nonlinear systems," IEEE Transactions on Systems, Man, and Cybernetics: Systems, vol. 51, no. 2, pp. 885-892, 2021.

[14] W. H. Qi, G. D. Zong, and W. X. Zheng, "Adaptive eventtriggered SMC for stochastic switching systems with semiMarkov process and application to boost converter circuit model," IEEE Transactions on Circuits and Systems I: Regular Papers, vol. 68, no. 2, pp. 786-796, 2020.

[15] S. H. Huang and J. Wang, "Fixed-time fractional-order sliding mode control for nonlinear power systems," Journal of Vibration and Control, vol. 26, no. 17, pp. 1425-1434, 2020.

[16] V. V. Huynh, B. L. N. Minh, E. N. Amaefule, A.-T. Tran, and P. T. Tran, "Highly robust observer sliding mode based frequency control for multi area power systems with renewable power plants," Electronics, vol. 10, no. 3, pp. 274-294, 2021.

[17] N. K. Singh and V. Mahajan, "Analysis and evaluation of cyber-attack impact on critical power system infrastructure," Smart Science, vol. 9, no. 1, pp. 1-13, 2021.

[18] X.-C. ShangGuan, Y. He, C.-K. Zhang et al., "Switching system-based load frequency control for multi-area power system resilient to denial-of-service attacks," Control Engineering Practice, vol. 107, pp. 104678-104689, 2021.

[19] L. Zhao and W. Li, "Co-design of dual security control and communication for nonlinear CPS under DoS attack," IEEE Access, vol. 8, pp. 19271-19285, 2020.

[20] B. Hu, H. Wang, Y. Zhao et al., "A brief overview of optimal robust control strategies for a benchmark power system with different cyber-physical attacks," Complexity, vol. 2021, Article ID 6646799, 2021.

[21] W. Qi, Y. Hou, G. Zong, and C. K. Ahn, "Finite-time eventtriggered control for semi-Markovian switching cyberphysical systems with FDI attacks and applications," IEEE Transactions on Circuits and Systems I: Regular Papers, vol. 68, no. 6, pp. 2665-2674, 2021.

[22] Z. H. Pang and G. P. Liu, "Design and implementation of secure networked predictive control systems under deception attacks," IEEE Transactions on Control Systems Technology, vol. 20, no. 5, pp. 1334-1342, 2011.

[23] M. R. Habibi, H. R. Baghaee, T. Dragievi et al., "False data injection cyber-attacks mitigation in parallel DC/DC converters based on artificial neural networks," IEEE Transactions on Circuits and Systems II: Express Briefs, vol. 68, no. 2, pp. 717-721, 2020.

[24] D. Du, R. Chen, X. Li, L. Wu, P. Zhou, and M. Fei, "Malicious data deception attacks against power systems: a new case and its detection method," Transactions of the Institute of Measurement and Control, vol. 41, no. 6, pp. 1590-1599, 2019.

[25] Y. Li, P. Zhang, L. Zhang et al., "Active synchronous detection of deception attacks in microgrid control systems," IEEE Transactions on Smart Grid, vol. 8, no. 1, pp. 373-375, 2016.

[26] R. C. B. Hink, J. M. Beaver, M. A. Buckner et al., "Machine learning for power system disturbance and cyber-attack discrimination," in Proceedings of the 7th International Symposium on Resilient Control Systems IEEE, pp. 1-8, Denver, CO, USA, September 2014.

[27] D. Zhao, Z. Wang, G. Wei, and Q.-L. Han, "A dynamic eventtriggered approach to observer-based PID security control subject to deception attacks," Automatica, vol. 120, pp. 109128-109141, 2020.
[28] J. Shi, "Cooperative control for nonlinear multi-agent systems based on event-triggered scheme," IEEE Transactions on Circuits and Systems II: Express Briefs, vol. 68, no. 6, pp. 1977-1981, 2020.

[29] E. Tian and C. Peng, "Memory-based event-triggering $\mathrm{H} \infty$ load frequency control for power systems under deception attacks," IEEE Transactions on Cybernetics, vol. 50, no. 11, pp. 4610-4618, 2020.

[30] D. Wang, F. Chen, B. Meng, X. Hu, and J. Wang, "Event-based secure Ho load frequency control for delayed power systems subject to deception attacks," Applied Mathematics and Computation, vol. 394, no. 1, pp. 125788-125801, 2021.

[31] W. Shen, S. Liu, and M. Liu, "Adaptive sliding mode control of hydraulic systems with the event trigger and finite-time disturbance observer," Information Sciences, vol. 569, pp. 55-69, 2021.

[32] M. M. Hossain and C. Peng, "Observer-based event triggeringHoLFC for multi-area power systems under DoS attacks,” Information Sciences, vol. 543, pp. 437-453, 2021.

[33] D. Yue, E. Tian, and Q.-L. Han, "A delay system method for designing event-triggered controllers of networked control systems," IEEE Transactions on Automatic Control, vol. 58, no. 2, pp. 475-481, 2013.

[34] S. Boyd, L. El Ghaoui, E. Feron et al., Linear Matrix Inequalities in System and Control Theory, SIAM, Philadelphia, PA, USA, 1994. 